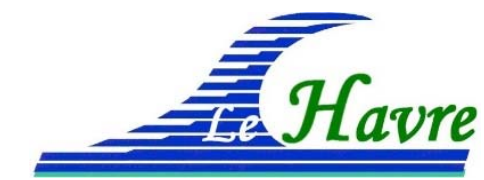

\title{
Impact des activités humaines sur l'évolution du littoral en Afrique de l'Ouest - Contribution des données historiques de WACA-F
}

\author{
Manuel GARCIN ${ }^{1}$, Clément GICQUEL ${ }^{1,2}$, Julie BILLY ${ }^{1}$, \\ Franck DESMAZES ${ }^{1}$, Aurélie MASPATAUD ${ }^{1}$
}

\author{
1. BRGM DRP/R3C, 3 avenue Claude Guillemin, BP 36009, 45060 Orléans Cedex 2, \\ France. \\ m.garcin@brgm.fr \\ 2. SHOM, 13 rue du Châtellier, 29200 Brest, France.
}

\section{Résumé :}

Des sites comme Saint-Louis (Sénégal), Cotonou (Bénin), Lomé (Togo) ou Grand-Popo (site transfrontalier Togo-Bénin) sont généralement considérés comme emblématiques de l'érosion côtière affectant l'Ouest africain. La mise en danger de la population et de forts enjeux sociaux-économiques posent des problèmes aux autorités en charge de la gestion du littoral. Par ailleurs, les prévisions du changement climatique et la remontée du niveau marin associée, sont sources d'une inquiétude légitime pour les populations de ces zones basses. Une restitution de l'évolution des côtes du Sénégal, du Togo et du Bénin a été réalisée à partir de données historiques issues des archives de l'IGN et du SHOM (projet WACA-F ; financement MTES). L'analyse des traits de côte sur plusieurs décennies (de 1954/1955 à aujourd'hui) confirme la mobilité du littoral sur ces territoires. Toutefois, la mise en perspective de ces évolutions avec : (i) les forçages météo-marins, (ii) l'évolution de l'occupation du sol, (iii) la construction d'ouvrages (portuaires, de protection côtière, de barrage sur les fleuves) permettent de déterminer la multiplicité des causes. Il apparait clairement que pour les côtes togolaise et béninoise, les zones où le recul du trait de côte est le plus critique sont directement liées à l'implantation d'ouvrages portuaires ayant perturbé la dynamique hydro-sédimentaire locale (jetées, digues, épis, etc.). Sur d'autres sites comme Saint-Louis, les effets négatifs sont liés à l'augmentation des surfaces artificialisées sur le cordon sableux (11\% en 1954, 40\% en 2018) et corrélativement à la fixation par des ouvrages de cette zone naturellement mobile. De plus, ces littoraux subissent un déficit d'apport sédimentaire par les fleuves induit par le piégeage de sédiments dans les barrages. Enfin, dans d'autres cas, les déséquilibres sont directement liés à des interventions humaines sur les flèches sableuses (création de brèche pour l'évacuation des crues) qui modifient profondément la dynamique hydro-sédimentaire. Les craintes liées aux effets de la remontée du niveau marin sur ces littoraux, dans des contextes où leurs résiliences naturelles ont été amoindries, sont d'autant plus justifiées. Mots-clés : Trait de côte, Occupation du sol, Anthropisation, Artificialisation, Environnement littoral, Changement climatique, Atlantique, Afrique de l'Ouest. 


\section{Thème 6 - Gestion durable des zones littorales et estuariennes}

\section{Introduction}

Les pays d'Afrique de l'Ouest sont actuellement confrontés à une érosion côtière et à un recul du trait de côte qui leur sont préjudiciables. Dans ce contexte, le projet WACA-F (financement MTES, coordination CEREMA) vise à mettre à disposition du Sénégal, du Togo et du Bénin les données acquises historiquement par le SHOM (de 1885-2006: près de 243 cartes et minutes papier) et l'IGN (7 800 photographies aériennes couvrant, selon les localisations les années 1954, 1955, 1969, 1977), voir figure 1. Les littoraux de ces trois pays sont caractérisés par une puissante dérive littorale assurant un fort transit sédimentaire. Les côtes du Sénégal sont par ailleurs exposées à de fortes houles de NW tandis que les vagues affectant les côtes du Togo et du Bénin sont moins énergétiques. La numérisation et la mise aux standards actuels des données historiques permet de constituer un socle de référence pour la compréhension à long terme de l'évolution du littoral ouest-africain. Cet article présente quelques résultats issus de leur analyse et les enseignements que l'on peut en tirer. Des recherches ont déjà été réalisées sur certains secteurs des trois pays concernés; les données issues du projet WACA permettent de confirmer certains résultats et de restituer l'évolution du littoral sur une durée de 62 ans.
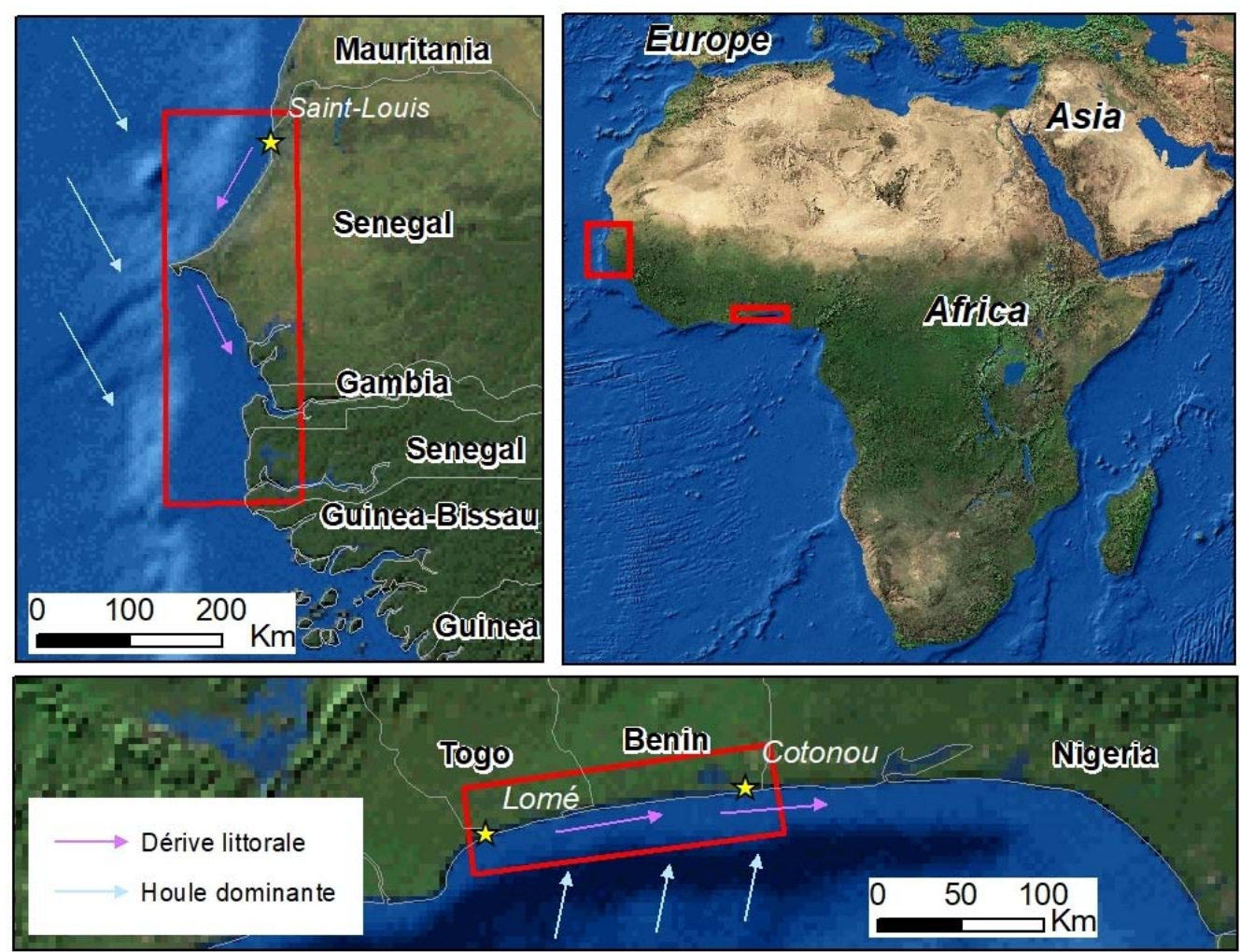

Figure 1. Localisation et contextes des zones traitées dans le projet WACA-F. 


\section{XVI'mes Journées Nationales Génie Côtier - Génie Civil \\ Le Havre 2020}

\section{Méthode}

\subsection{Numérisation des données historiques}

Les travaux de numérisation des photographies aériennes anciennes, de géo référencement et la création des ortho photographies ont été réalisés par l'IGN (résolution de 1,5 m). La numérisation et le géo référencement des données bathymétriques anciennes (dont certaines datent du XIXème siècle) ont été effectuées par le SHOM.

\subsection{Interprétation des ortho photographies : les indicateurs du trait de côtes}

Plusieurs indicateurs du trait de côte (morphologiques, hydrologiques ou biologiques ; BOAK \& TURNER, 2005) ont été cartographiés sur les trois pays:

a) Limite du jet de rive - marquant la position du bas de la plage subaérienne ou de la zone intertidale.

b) Limite de végétation - assimilable à la limite haute de la plage subaérienne (FAYE, 2010; MOORE, 2000).

c) Limite d'urbanisation - limite des zones bâties côté mer qui constitue généralement l'indicateur de haut de plage.

d) Limite mixte - dans les zones d'imbrication de l'urbanisation et de secteur naturel, indicateur de haut de plage a été qualifié de mixte.

e) Limite des ouvrages - Quand le littoral est bordé par une route, un perré, une digue, un quai etc., c'est la limite de l'ouvrage côté mer qui a été prise comme indicateur du trait de côte. En fonction de la complexité des côtes ces indicateurs ont été digitalisés à une échelle comprise entre 1/3000 et 1/1500 sur les ortho-mosaïques anciennes (tableau 1) et sur les images satellite (2017). Pour chaque segment de trait de côte, l'indicateur choisi et son appartenance au groupe "naturel" ou "anthropisé" ont été renseignés.

\subsection{Analyse diachronique des traits de côte}

L'analyse diachronique des traits de côte a été réalisée sous ArcGis, à l'aide de l'extension DSAS (Digital Shoreline Analysis System v5.0 de l'USGS) selon la méthode écrite par HIMMELSTOSS et al., (2018). Une analyse par transects espacés de $50 \mathrm{~m}$ a été réalisée par exemple sur les $170 \mathrm{~km}$ constituant les côtes togolaise et béninoise (3400 transects). Une analyse a ensuite été conduite afin d'extraire du traitement automatique les secteurs (embouchures, zones de brèches, flèches mobiles...) où cette approche n'est pas pertinente.

\subsection{Analyse diachronique de l'occupation du sol}

Une analyse de l'évolution de l'occupation du sol a été conduite sur certains secteurs (par exemple Langue de Barbarie) afin de déterminer comment l'occupation du sol a évolué depuis les années 1950 et de comprendre son rôle dans l'évolution du trait de côte. Plusieurs grandes classes d'occupation du sol ont été identifiées: sols nus sableux - zones 


\section{Thème 6 - Gestion durable des zones littorales et estuariennes}

sableuses à végétation éparse - zones à végétation dense (mangrove comprises) - zones urbanisées ou artificialisées - fleuve et continent (première terre détectée à l'est après le fleuve). La cartographie a été réalisée à chaque date à une échelle comprise entre 1/2000 à $1 / 1500$ sous ArcGis (C ESRI). Une analyse par transects espacés de $50 \mathrm{~m}$ des différentes classes d'occupation du sol a été réalisée via l'outil DSAS. Elle a permis de déterminer les largeurs occupées par chaque classe d'occupation du sol présentes sur le cordon et d'en mesurer l'évolution dans le temps. 840 transects ont ainsi été réalisés sur la Langue de Barbarie. Ils ont permis de réaliser des statistiques de largeur de nature d'occupation du sol par date.

\section{Résultats}

Les différents indicateurs du trait de côte ont été cartographiés sur les $730 \mathrm{~km}$ du littoral sénégalais (1954, 2017), les $50 \mathrm{~km}$ du Togo et les $120 \mathrm{~km}$ du Bénin (1955, 2017). Les ortho photographies de 1969 et de 1977 de la côte togolaise ont permis de compléter l'analyse à des dates intermédiaires. La côte togolaise voit ainsi son artificialisation passer de $11 \%$ en 1955 à $57 \%$ en 2017 avec une évolution très rapide sur la période 1955-1969 (figure 2). L'artificialisation de la côte béninoise quant à elle passe de $9,5 \%$ à près de $51 \%$ de 1955 à 2017.
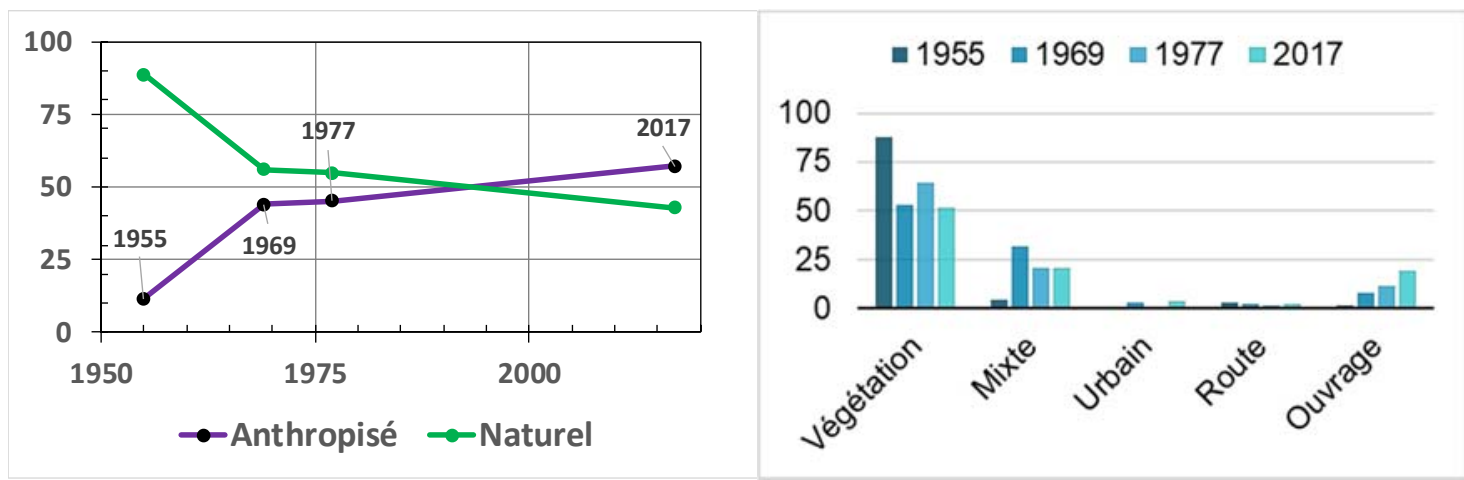

Figure 2. Nature du littoral du Togo en 1955, 1969, 1977 et 2017 - A gauche rapport côte naturelle versus anthropisée, à droite nature du trait de côte de haut de plage (pourcentage de longueur de côte par type).

L'analyse diachronique (1955-2017) permet de restituer l'évolution du trait de côte à partir de données homogènes à l'échelle des pays concernés sur 62 ans (tableau 1, figure 3 ). On constate un recul généralisé du trait de côte (13m de recul moyen pour les deux pays) confirmant les résultats d'études antérieures plus ponctuelles (BLIVI \& ADJOUSSI, 2004). Localement des valeurs (d'avancée et de recul) peuvent être très fortes (jusqu'à $+1121 \mathrm{~m}$ et $-690 \mathrm{~m}$ ). Ces zones les plus mobiles sont toutes associées à des perturbations anthropiques. 


\section{XVIèmes Journées Nationales Génie Côtier - Génie Civil \\ Le Havre 2020}

\section{Discussion}

Les analyses présentées précédemment ont permis d'identifier plusieurs types d'actions anthropiques à fort impact:

i) urbanisation et constructions en dur sur des zones naturellement mobiles (ex. SaintLouis-du-Sénégal),

ii) édification d'infrastructures (ex. ports de Cotonou et de Lomé), iii) réalisation de brèches volontaires dans les cordons sableux (ex. Langue de Barbarie au Sénégal en 2003 ; Bouche du Roi au Bénin en 1999 et en 2009 ; LAIBI, 2010 ; NDOUR et al., 2018).

A ces causes, il faut ajouter d'autres actions ayant affecté le bilan sédimentaire littoral:

i) l'édification de barrages sur les fleuves qui ont modifiés, d'une part, les régimes fluviaux et, d'autre part, diminué les apports sédimentaires (BLIVI, 2000; GIARDINO et al., 2018 ; AMOUSSOU, 2010),

ii) des actions plus locales à effet cumulatif tel que les prélèvements de granulats sur les plages. Ces modifications hydrologiques et sédimentaires amplifient les effets induits par les perturbations ponctuelles du littoral.

Tableau 1. Évolution du trait de côte du Togo et du Bénin de 1955 à 2017.

\begin{tabular}{llllll}
\hline $\begin{array}{l}\text { Évolution du } \\
\text { trait de côte }\end{array}$ & $\begin{array}{l}\text { Évolution } \\
\text { moyenne }\end{array}$ & $\begin{array}{l}\text { Mobilité } \\
\text { moyenne }\end{array}$ & $\begin{array}{l}\text { Recul } \\
\text { maximum }\end{array}$ & $\begin{array}{l}\text { Avancée } \\
\text { maximum }\end{array}$ & $\begin{array}{l}\text { Longueur } \\
\text { littoral }\end{array}$ \\
\hline Togo \& Bénin & $-13 \mathrm{~m}$ & $-0.2 \mathrm{~m} / \mathrm{an}$ & $-690 \mathrm{~m}$ & $1121 \mathrm{~m}$ & $170 \mathrm{~km}$ \\
\hline Togo & $-29 \mathrm{~m}$ & $-0.5 \mathrm{~m} / \mathrm{an}$ & $-342 \mathrm{~m}$ & $1066 \mathrm{~m}$ & $50 \mathrm{~km}$ \\
\hline Bénin & $-7 \mathrm{~m}$ & $-0.1 \mathrm{~m} / \mathrm{an}$ & $-690 \mathrm{~m}$ & $1121 \mathrm{~m}$ & $120 \mathrm{~km}$ \\
\hline
\end{tabular}

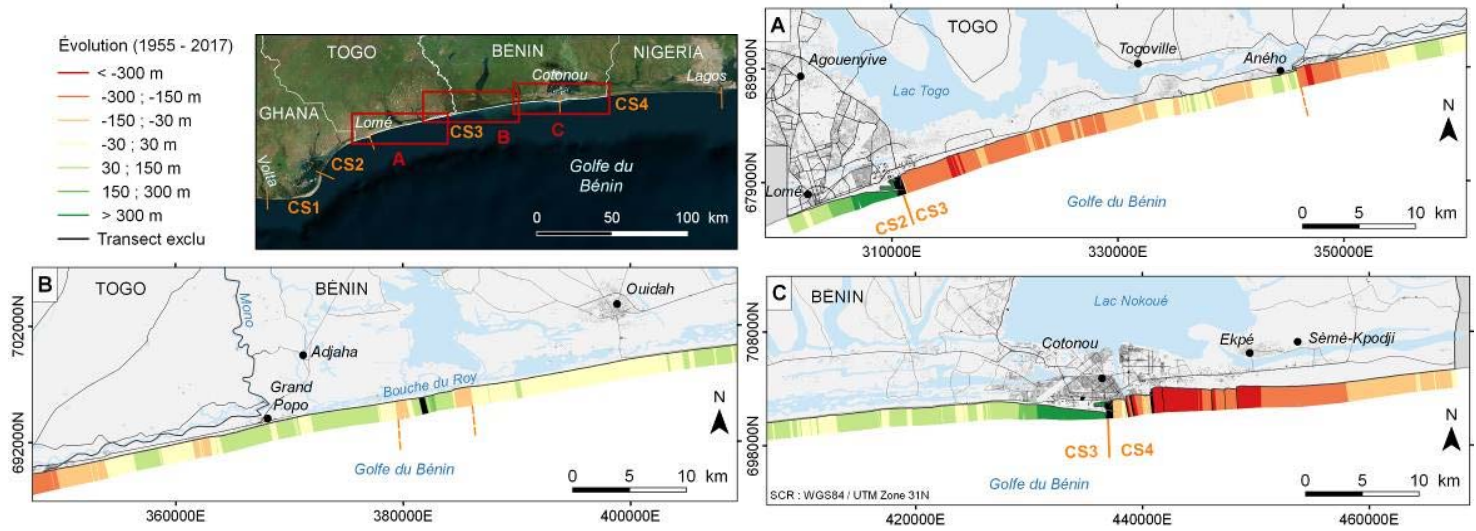

Figure 3. En haut les différentes cellules sédimentaires (CS1 à CS4) du Golfe du Bénin - Encarts A à C : évolution (en m) du trait de côte entre 1955 et 2017. 


\section{Thème 6 - Gestion durable des zones littorales et estuariennes}

\subsection{Impact des grandes infrastructures sur le long terme : exemple du port de Lomé (Togo)}

Les jetées portuaires ont des effets opposés sur la mobilité du trait de côte entre l'amontdérive et l'aval-dérive de l'ouvrage. Les distances sur lesquelles les ouvrages ont des impacts significatifs sont évolutives en fonction de la durée écoulée depuis leur installation. Le tableau 2 illustre l'évolution temporelle des impacts du Port de Lomé, mis en service en 1968. On y observe : (i) dès 1969 les effets sur l'évolution du trait de côte sont très importants, (ii) que la dissymétrie amont-aval est forte et apparaît très précocement, et qu'enfin (iii) les perturbations induites se propagent en fonction du temps. Toutefois la perturbation en amont-dérive semble se stabiliser au cours du temps, alors que la perturbation en aval-dérive continue à se propager. Actuellement, les distances impactées par les effets induits par la jetée du port de Lomé atteignent au maximum $8 \mathrm{~km}$ en amont dérive et $22 \mathrm{~km}$ en aval dérive. Les avancées et les reculs du trait de côte maximaux sont observés à proximité immédiate de l'ouvrage (ex. progradation maximum de l'ordre de $700 \mathrm{~m}$ et recul maximum de l'ordre de $1120 \mathrm{~m}$ en 62 ans à Cotonou).

4.2 Impact de l'urbanisation et de l'artificialisation des flèches sableuses : l'exemple de Saint-Louis-du-Sénégal

Certains quartiers de Saint-Louis au Nord du Sénégal (Goxy Mbathie, Get N’r), sont implantés sur la flèche sableuse de la Langue de Barbarie. Cette flèche délimite le fleuve Sénégal à l'Est et est exposée aux puissantes houles atlantiques de secteur NW (Hs : 1,6 m, période : $14 \mathrm{~s}$; SY, 2010). Une dérive littorale puissante induit un transport sédimentaire évalué aux alentours de $5 \times 10^{5} \mathrm{~m}^{3} /$ an (SADIO et al., 2017). Ces quartiers, et plus particulièrement la première ligne de bâtiments côté océan, ont été partiellement à totalement détruits au cours des cinq dernières années par l'action des vagues. Ce site est généralement considéré, notamment par les médias, comme emblématique de l'érosion du littoral résultant de l'impact du changement climatique. Les analyses réalisées à partir des données historiques montrent que l'érosion existe effectivement mais qu'elle est relativement modeste quand on la compare aux reculs observés par ailleurs (tableau 1). La comparaison des indicateurs du trait de côte entre les années 1954 et 2017 montre, en effet, que le recul du trait de côte sur le Nord de la Langue de Barbarie est contrasté (figure 4) : au nord de Saint Louis dans le secteur naturel, on observe une translation d'une cinquantaine de mètres du cordon vers l'Est ; plus au Sud, un quartier s'est installé après 1954 sur le haut de plage et l'ensemble du cordon. On y observe un recul de l'indicateur "bas-de-plage" jusqu'à se confondre avec celui du haut de plage qui est, lui, figé par les constructions ; la plage a disparu. Dans les quartiers plus au sud et plus anciens, on note la même évolution (coalescence des 2 indicateurs, disparition de la plage). Dans ces deux cas, la première ligne de bâti se retrouve alors directement exposée aux vagues qui déferlent sur les bâtiments et qui causent leur destruction. Au sud des zones construites, 


\section{XVIèmes Journées Nationales Génie Côtier - Génie Civil \\ Le Havre 2020}

la plage s'élargit à nouveau, les indicateurs du trait de côte restent quasi-stables entre 1954 et 2017. Ceci nous indique :

1) que le transport par la dérive Nord-Sud et les apports sédimentaires, bien qu'ayant probablement diminué sur les dernières années, sont restés suffisants pour que la plage se maintienne ;

2) que la disparition de la plage et les dégâts observés sont à mettre en relation avec l'artificialisation du cordon, le blocage de sa libre évolution et la présence d'enjeux dans un secteur naturellement dynamique et mobile.

La capacité d'adaptation du littoral et la résilience de ce secteur ont été fortement altérées par la présence du bâti (murs, habitations, etc.). La situation actuelle d'exposition au risque est en grande partie héritée de choix d'aménagement et d'un développement urbain qui ne sont pas compatibles avec la dynamique naturelle de ce littoral.

Tableau 2. Distances où les impacts des jetées du Port de Lomé affectent les processus sédimentaires.

\begin{tabular}{llllll}
\hline $\begin{array}{l}\text { Distances en } \mathbf{k m} \text { de côte } \\
\text { impactée / port de Lomé }\end{array}$ & $\mathbf{1 9 5 5}$ & $\mathbf{1 9 6 8}$ & $\mathbf{1 9 6 9}$ & $\mathbf{1 9 7 7}$ & $\mathbf{2 0 1 7}$ \\
\hline Amont dérive & - & Mise en & 3,5 & 7,7 & 8 \\
\cline { 5 - 7 } Aval dérive & - & $\begin{array}{l}\text { service } \\
\text { du port }\end{array}$ & 8 & 14 & 22 \\
\hline
\end{tabular}

4.3 Impact des ouvertures de brèches artificielles dans les flèches sableuses : exemple de la Brèche de la Langue de Barbarie (Sénégal)

Afin de faciliter les écoulements lors des crues des fleuves menaçant certaines villes, des brèches artificielles ont été réalisées dans plusieurs flèches (Langue de Barbarie au Sénégal, Aného au Togo, Bouche du Roi au Bénin). La Langue de Barbarie est un cordon sableux entretenu par une dérive littorale Nord-Sud délimitant le fleuve Sénégal. La migration vers le Sud de cette flèche conditionne la position de l'embouchure du Sénégal. En 2003 une brèche a été réalisée dans la flèche à $23 \mathrm{~km}$ en amont de l'embouchure. Cette brèche est devenue le nouvel exutoire du Sénégal ce qui engendre une rupture du transport sédimentaire entre le Nord et le Sud de la brèche (effet de chasse). La bordure nord de la brèche est devenue l'extrémité de la flèche sableuse dont la progression se poursuit au rythme de $300 \mathrm{~m} / \mathrm{an}$ vers le sud. En revanche, la partie située au sud de la brèche est déconnectée de son apport sédimentaire ce entraine son érosion à une vitesse bien supérieure. Un élargissement très rapide de la largeur de la brèche s'est alors produit pour atteindre 4,7 km en 2019. Celui-ci est la cause de l'érosion intense des îles et des cordons formant la rive droite du Sénégal. Ils étaient auparavant protégés de la dynamique marine par la flèche et y sont dorénavant exposés. La destruction des villages et des plantations qui étaient établis sur ces îles et ces cordons ont été très préjudiciables pour les populations. 


\section{Thème 6 - Gestion durable des zones littorales et estuariennes}

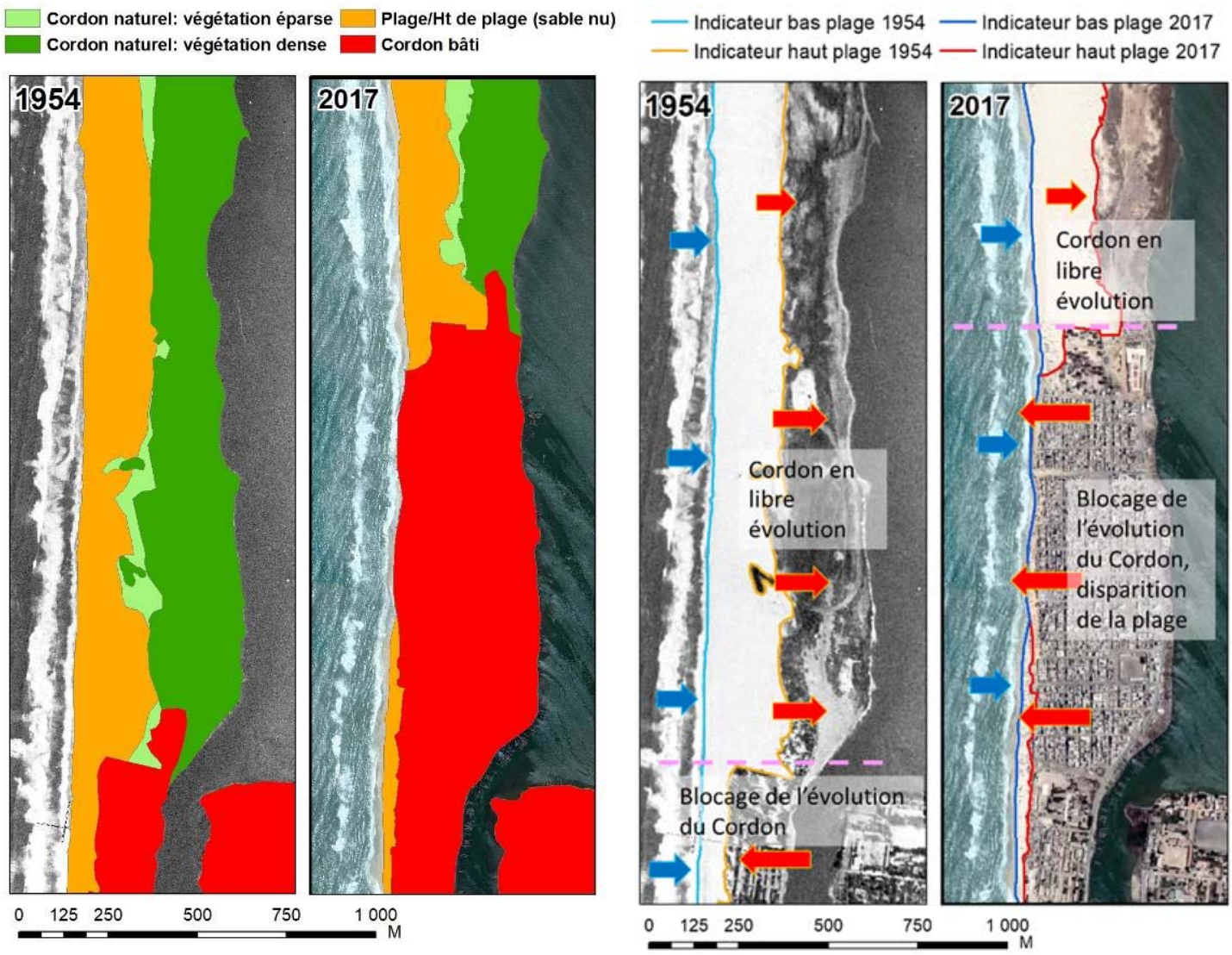

Figure 4. Evolution de l'occupation du sol à Saint-Louis nord entre 1954 et 2017 Incidence de l'artificialisation sur l'évolution de la Langue de Barbarie.

\section{Conclusions}

Les travaux réalisés à l'échelle du Sénégal, du Togo et du Bénin confirment une tendance au recul du trait de côte de 1954 à 2017. Toutefois, les secteurs où les évolutions du trait de côte sont les plus spectaculaires et où les impacts sur les enjeux (humains et économiques) sont les plus importants sont ceux où des infrastructures et des actions humaines ont perturbé significativement la dynamique côtière. Les options d'aménagement et de gestion du littoral de ces zones à risque devront prendre en compte leurs dynamiques particulières afin d'être durables. Les craintes liées aux effets de la remontée du niveau marin, dans des contextes où la résilience naturelle du littoral a été amoindrie par les actions anthropiques, sont d'autant plus justifiées. 


\section{XVİ̀mes Journées Nationales Génie Côtier - Génie Civil \\ Le Havre 2020}

\section{Références}

AMOUSSOU E. (2010). Variabilité pluviométrique et dynamique hydro-sédimentaire du bassin versant du complexe fluvio-lagunaire Mono-Ahémé-Couffo (Afrique de l'ouest).Thèse de doctorat, Université de Bourgogne, $313 \mathrm{p}$.

BLIVI A.B. (2000). Effets du barrage de Nangbéto sur l'évolution du trait de côte : une analyse prévisionnelle sédimentoloique. Journal de la Recherche Scientifique de l'Université du Bénin (Togo), Vol. 4, pp 29-41. https://doi.org/10.4314/jrsul.v4i1.16996

BLIVI A.B., ADJOUSSI P. (2004). La cinématique du trait de côte au Togo vue par télédétection (Moving of the Togo shoreline detected by remote sensing), Geo-Eco-Trop, Vol. 28(1-2), pp 27-38.

BOAK E.H., TURNER I.L. (2005). Shoreline definition and detection: A review. Journ. Coast. Res., Vol. 21, 4, pp 688-703, https://doi.org/10.2112/03-0071.1

FAYE I. B. N. (2010). Dynamique du trait de côte sur les littoraux sableux de la Mauritanie à la Guinée-Bissau (Afrique de l'Ouest): Approches régionale et locale par photo-interprétation, traitement d'images et analyse de cartes anciennes. Thèse de doctorat de l'Université de Bretagne Occidentale, $322 \mathrm{p}$.

GIARDINO A., SCHRIJVERSHOF R., NEDERHOFF C.M., DE VROEG H., BRIERE C., TONNON P.-K., CAIRES S., WALSTRA D.J., SOSA J., VAN VERSEVELD W., SCHELLEKENS J., SLOFF C.J. (2018). A quantitative assessment of human interventions and climate change on the West African sediment budget. Ocean Coast. Manag., Vol. 156, pp 249-265. https://doi.org/10.1016/j.ocecoaman.2017.11.008

HIMMELSTOSS E.A., HENDERSON R.E., KRATZMANN M.G., FARRIS A.S. (2018). Digital Shoreline Analysis System (DSAS) version 5.0 user guide (USGS Numbered Series No. 2018-1179), Open-File Report. U.S. Geological Survey, Reston, VA. https://doi.org/10.3133/ofr20181179

LAIBI R. (2010). Les plages béninoises dans le golfe de Guinée en Afrique de l'Ouest: Evolutions et conséquences socio-économiques. Uemoa - SDLAO, 6M, 58.

MOORE L.J. (2000). Shoreline Mapping Techniques. J. Coast. Res. Vol. 16(1), pp 111-124.

NDOUR A., LAÏBI R. A., SADIO M., DEGBE C. G. E., DIAW A. T., OYEDE L. M., ANTHONY E. J., DUSSOUILlEZ P., SAMBOU H., DIÈYE E. H. B. (2018). Management strategies for coastal erosion problems in west Africa: Analysis, issues, and constraints drawn from the examples of Senegal and Benin. Ocean and Coastal Management, Vol. 156, pp 92-106. https://doi.org/10.1016/j.ocecoaman.2017.09.001

SADIO M., ANTHONY E.J., DIAW A.T., DUSSOUILLEZ P., FLEURY J.T., KANE A., ALMAR R., KESTENARE E. (2017). Shoreline Changes on the Wave-influenced Senegal river delta, West Africa: The roles of natural processes and human interventions. Water, Vol. 9(5), 357. https://doi.org/10.3390/w9050357

SY A. B. (2010). Etude de la dynamique du cordon littoral du gandiolais sur l'axe Saint louis - Niayam 2010. In Uemoa - SDLAO, (Vol. 6B). 
Thème 6 - Gestion durable des zones littorales et estuariennes 